\title{
On Fiducial Target Registration Error in the Presence of Anisotropic Noise
}

\author{
Burton Ma ${ }^{1}$, Mehdi H. Moghari ${ }^{2}$, Randy E. Ellis ${ }^{1,3}$, and Purang Abolmaesumi ${ }^{1,2,3}$ \\ ${ }^{1}$ Human Mobility Research Centre, Kingston General Hospital, Kingston, Ontario, Canada \\ ${ }^{2}$ Department of Electrical Engineering, Queen's University, Kingston, Ontario, Canada \\ ${ }^{3}$ School of Computing, Queen's University, Kingston, Ontario, Canada \\ mab, hedjazi, ellis, purang@cs.queensu.ca
}

\begin{abstract}
We study the effect of anisotropic noise on target registration error (TRE) by using a tracked and calibrated stylus tip as the fiducial registration application. We present a simple, efficient unscented Kalman filter algorithm that is suitable for fiducial registration even with a small number of fiducials. We also derive an equation that predicts TRE under anisotropic noise. The predicted TRE values are shown to closely match the simulated TRE values achieved using our UKF-based algorithm.
\end{abstract}

\section{Introduction}

Many least-squares solutions have been proposed for the problem of fiducial (pairedpoint) registration. The use of least-squares assumes that one set of points is noise free and the other set of points is contaminated with isotropic, zero mean, independent, identically distributed (iid) Gaussian noise.

Optical tracking systems that sense points of infrared light are commonly used in commercial navigated surgical systems. These systems measure coordinate reference frames (CRFs), which are essentially a set of infrared emitting/reflecting fiducial markers, that are rigidly attached to the tracked object. The measurement precision is typically worse in the viewing direction of the cameras for such tracking systems. Khadem and colleagues [1] found that the jitter in the measured position of a static target was anisotropic with the greatest deviation occurring in the viewing direction of the tracking system. Their results showed an anisotropy as large as a factor of five or more when using a Polaris tracking system with a passive target.

Ohta and Kanatani [2] described an algorithm designed to accommodate anisotropic, non-identical Gaussian noise in both the model and measurement coordinate systems. This algorithm was used in a modified version of the ICP algorithm [3].

Pennec and Thirion [4] used an extended Kalman filter as part of a framework for registration using points and frames. Their approach accommodated anisotropic noise in both sets of points to be registered.

Fitzpatrick and colleagues [5] derived an expression for fiducial target registration error (TRE) in $k$-dimensions. Target registration error is simply the magnitude $\| \mathbf{r}-$ $\mathbf{r}^{\prime} \|$ where $\mathbf{r}$ is the expected location of a target point and $\mathbf{r}^{\prime}$ is the registered location the target point. Their derivation was performed assuming zero-mean, isotropic, iid 
Gaussian noise. West and Maurer [6] described how to use these results to design targets for optically tracked surgical instruments.

Ma and Ellis [7] presented analytic expressions based on a spatial stiffness model for TRE for both fiducial and surface-based registration. Their expression for fiducial TRE was identical to that published by [5]; thus, their approach was only applicable to isotropic Gaussian iid noise. It can be shown that the stiffness matrices they derived are based on first-order Taylor series approximations of rotation and translation [8].

Moghari and Abolmaesumi [9] used the unscented Kalman filter (UKF) to solve the fiducial registration problem and estimate the covariance of the state parameters $\left[t_{x}, t_{y}, t_{z}, \theta_{x}, \theta_{y}, \theta_{z}\right]^{T}$, where $\left[t_{x}, t_{y}, t_{z}\right]^{T}$ is the translation and $\left[\theta_{x}, \theta_{y}, \theta_{z}\right]^{T}$ is the vector of $Z Y X$ Euler angles. Given a sufficient number of markers, their algorithm was able to estimate the mean squared TRE and the distribution of TRE. Their work appears to be an improvement over [4] with regard to estimating TRE and its distribution [10].

We present three significant contributions over prior art in this article. The first contribution is a comparison of fiducial registration algorithms when there is anisotropic, identically distributed noise in the fiducial measurements. The second contribution is the derivation of an equation that predicts the expected root mean square (RMS) TRE for fiducial registration with anisotropic noise. The third contribution is the introduction of a simple, UKF-based fiducial registration algorithm that, as we demonstrate, achieves the TRE predicted by our derived equation. We use simulations of a pointing stylus and an image registration problem to demonstrate the effects of anisotropic noise on TRE.

\section{Method}

\subsection{UKF Fiducial Registration Algorithm}

A conventional filtering algorithm processes observations as they are made available, and then does not reconsider them; in this way, it is able to efficiently perform a sequential update of the state estimate. The UKF algorithm described in [9] is unusual in that it continually reprocesses previous observations by appending new observations to the vector of old observations, lengthening the observation vector with each marker observation; this negates the advantage of efficient state estimate updates. Our UKF algorithm processes all fiducial marker observations in one update. The state model is $\mathbf{x}_{i+1}=\mathbf{x}_{i}+\mathbf{v}_{i}$ where $\mathbf{x}_{i}=\left[t_{x}, t_{y}, t_{z}, \theta_{x}, \theta_{y}, \theta_{z}\right]_{i}^{T}$ is the vector of registration parameters (identical to that in [9]) at time $i$ and $\mathbf{v}_{i}$ is the noise associated with the uncertainty of the state estimate. We assume that $\mathbf{v}_{i}$ is drawn from a zero-mean Gaussian with covariance matrix $\mathbf{V}_{i}$. Our observation model for $n$ fiducial markers is $\mathbf{y}_{i}=\left[\begin{array}{c}\mathbf{g}_{1} \\ \vdots \\ \mathbf{g}_{n}\end{array}\right]=\left[\begin{array}{c}\mathbf{R}\left(\theta_{x}, \theta_{y}, \theta_{z}\right)_{i} \mathbf{f}_{1}+\left[t_{x}, t_{y}, t_{z}\right]_{i}^{T} \\ \vdots \\ \mathbf{R}\left(\theta_{x}, \theta_{y}, \theta_{z}\right)_{i} \mathbf{f}_{n}+\left[t_{x}, t_{y}, t_{z}\right]_{i}^{T}\end{array}\right]+\mathbf{n}_{i}$ where $\mathbf{g}_{j}$ is the $j^{\text {th }}$ measured fiducial location, $\mathbf{f}_{j}$ is the $j^{\text {th }}$ model fiducial location, $\mathbf{R}\left(\theta_{x}, \theta_{y}, \theta_{z}\right)_{i}$ is the $Z Y X$ Euler rotation matrix computed using the estimated rotation state at time $i,\left[t_{x}, t_{y}, t_{z}\right]^{T}$ is the estimated translation at time $i$, and $\mathbf{n}_{i}$ is the measurement noise.

We can compute a good initial state estimate $\mathbf{x}_{0}$ using a least-squares algorithm such as Horn's method [11]. One iteration of the UKF is performed producing a new estimate of the registration parameters and parameter covariances. The estimate is accurate 
enough that it can be corrected by the filter even though Horn's method is known to be suboptimal under anisotropic noise.

We initialize the state parameter covariance $\mathbf{V}_{0}$ with a reasonable guess based on the expected measurement noise magnitude. All simulations in this article used a diagonal matrix for $\mathbf{V}_{0}$ with elements of $(1 \mathrm{~mm})^{2}$ for translation and $(1 \mathrm{rad})^{2}$ for rotation.

\subsection{Spatial Stiffness Analysis for Anisotropic Noise}

The model of fiducial registration published in [7] treated each noise-free fiducial location as an end point of a zero-length linear spring. Noise in the registered marker location was considered as a small extension of the spring. The springs had no preferred direction, which was appropriate for isotropic noise. We modify their model to accommodate anisotropic noise by replacing the single spring with three directional springs with appropriate spring constants. In general, the spring constants and directions are related to the principal components of the noise covariance matrix for the fiducial marker; the spring constants are the reciprocals of the eigenvalues of the covariance matrix, and the directions are the eigenvectors of the covariance matrix. If the covariance matrix is diagonal, then the spring constants are the reciprocals of the variances; that is, we weight the stiffness of each spring in inverse proportion to the noise variance just as we would in a typical weighted least-squares solution. For the purposes of this article, we will assume that we are working with fiducials measured in the optical tracker coordinate system so that the noise covariance matrix for the marker locations is diagonal.

Our derivation of the anisotropic stiffness matrix follows that of [7]. Let the $j^{\text {th }}$ fiducial be $\mathbf{f}_{j}=\left[x_{j}, y_{j}, z_{j}\right]^{T}$. If $\mathbf{f}_{j}$ is perturbed by a small rotation $\mathbf{R}\left(\theta_{x}, \theta_{y}, \theta_{z}\right)$ and translation $\mathbf{t}=\left[t_{x}, t_{y}, t_{z}\right]^{T}$, its new position is $\mathbf{g}_{j}=\mathbf{R}\left(\theta_{x}, \theta_{y}, \theta_{z}\right) \mathbf{f}_{j}+\mathbf{t}$. The potential energy stored in the springs associated with the marker can be written as $U_{j}=\frac{1}{2}\left(\mathbf{g}_{j}\right.$ $\left.\mathbf{f}_{j}\right)^{T} \operatorname{diag}\left(k_{x_{j}}, k_{y_{j}}, k_{z_{j}}\right)\left(\mathbf{g}_{j}-\mathbf{f}_{j}\right)$ where $\operatorname{diag}\left(k_{x_{j}}, k_{y_{j}}, k_{z_{j}}\right)$ is the $3 \times 3$ diagonal matrix of spring constants. The Hessian $\mathbf{H}_{j}$ of $U_{j}$ evaluated at zero displacement is

$$
\begin{aligned}
\mathbf{H}_{j}= & \mathbf{H}\left(U_{j} ; \theta_{x}=\theta_{y}=\theta_{z}=t_{x}=t_{y}=t_{z}=0\right) \\
& =\left[\begin{array}{cccccc}
k_{x_{j}} & 0 & 0 & 0 & k_{x_{j}} z_{j} & -k_{x_{j}} y_{j} \\
0 & k_{y_{j}} & 0 & -k_{y_{j}} z_{j} & 0 & k_{y_{j}} x_{j} \\
0 & 0 & k_{z_{j}} & k_{z_{j}} y_{j} & -k_{z_{j}} x_{j} & 0 \\
0 & -k_{y_{j}} z_{j} & k_{z_{j}} y_{j} & k_{z_{j}} y_{j}^{2}+k_{y_{j}} z_{j}^{2} & -k_{z_{j}} x_{j} y_{j} & -k_{y_{j}} x_{j} z_{j} \\
k_{x_{j}} z_{j} & 0 & -k_{z_{j}} x_{j} & -k_{z_{j}} x_{j} y_{j} & k_{z_{j}} x_{j}^{2}+k_{x_{j}} z_{j}^{2} & -k_{x_{j}} y_{j} z_{j} \\
-k_{x_{j}} y_{j} & k_{y_{j}} x_{j} & 0 & -k_{y_{j}} x_{j} z_{j} & -k_{x_{j}} y_{j} z_{j} & k_{y_{j}} x_{j}^{2}+k_{x_{j}} y_{j}^{2}
\end{array}\right]
\end{aligned}
$$

The stiffness matrix for $n$ markers is $\mathbf{K}=\sum_{j=1}^{n} \mathbf{H}_{j}=\left[\begin{array}{cc}\mathbf{A} & \mathbf{B} \\ \mathbf{B}^{T} & \mathbf{D}\end{array}\right]$ where $\mathbf{A}, \mathbf{B}$, and $\mathbf{D}$ are $3 \times 3$ block matrices. Following [7] we can write the mean squared TRE as

$$
\operatorname{TRE}^{2}(\mathbf{r}) \propto \underbrace{\frac{1}{\sigma_{1}}+\frac{1}{\sigma_{2}}+\frac{1}{\sigma_{3}}}_{\text {translational component }}+\underbrace{\frac{1}{\mu_{\mathrm{eq}, 1}}+\frac{1}{\mu_{\mathrm{eq}, 2}}+\frac{1}{\mu_{\mathrm{eq}, 3}}}_{\text {rotational component }}
$$

where $\mathbf{r}$ is the target location, $\sigma_{1}, \sigma_{2}, \sigma_{3}$ are called the principal translational stiffnesses and $\mu_{\mathrm{eq}, 1}, \mu_{\mathrm{eq}, 2}, \mu_{\mathrm{eq}, 3}$ are called the equivalent principal rotational stiffnesses. The stiffness quantities were first described by Lin and colleagues [12]; refer to [12] for details on computing the principal stiffnesses. It was previously shown that the rotational and 
translational stiffnesses are independent for fiducial registration [7]; thus, we are justified in using addition in quadrature of the two components of TRE in Equation 1 Only the rotational component of Equation 1 depends on the target location $\mathbf{r}$.

Equation (1) only gives the mean squared TRE to within a constant factor because the spring constants were scaled by the inverse of the variances. In the case of identical noise in all of the fiducials, we can recover the constant factor because the magnitude of the translational component can be computed in a different way. Let the identically distributed, zero-mean noise have covariance matrix $\mathbf{N}=\operatorname{diag}\left(n_{x}^{2}, n_{y}^{2}, n_{z}^{2}\right)$. Let the mean of the $n$ noisy marker locations be $\overline{\mathbf{F}}=\left[\bar{F}_{x}, \bar{F}_{y}, \bar{F}_{z}\right]^{T}$. The mean of the noise-free marker locations is the expected value $E[\overline{\mathbf{F}}]$. The expected squared translation magnitude of the mean marker location is simply the expected magnitude of $\overline{\mathbf{F}}-\mathrm{E}[\overline{\mathbf{F}}]$

$$
\begin{aligned}
\mathrm{E}\left[\delta^{2}\right] & =\mathrm{E}\left[\|\overline{\mathbf{F}}-\mathrm{E}[\overline{\mathbf{F}}]\|^{2}\right] \\
& =\mathrm{E}\left[\left(\bar{F}_{x}-\mathrm{E}\left[\bar{F}_{x}\right]\right)^{2}\right]+\mathrm{E}\left[\left(\bar{F}_{y}-\mathrm{E}\left[\bar{F}_{y}\right]\right)^{2}\right]+\mathrm{E}\left[\left(\bar{F}_{z}-\mathrm{E}\left[\bar{F}_{z}\right]\right)^{2}\right] \\
& =\operatorname{var}\left(\bar{F}_{x}\right)+\operatorname{var}\left(\bar{F}_{y}\right)+\operatorname{var}\left(\bar{F}_{z}\right) \\
& =\frac{1}{n}\left(n_{x}^{2}+n_{y}^{2}+n_{z}^{2}\right) .
\end{aligned}
$$

Equation (1) can now be rewritten to give the estimated squared TRE as

$$
\operatorname{TRE}^{2}(\mathbf{r})=f\left(\frac{1}{\sigma_{1}}+\frac{1}{\sigma_{2}}+\frac{1}{\sigma_{3}}+\frac{1}{\mu_{\text {eq }, 1}}+\frac{1}{\mu_{\text {eq }, 2}}+\frac{1}{\mu_{\text {eq }, 3}}\right), \quad f=\frac{\frac{1}{n}\left(n_{x}^{2}+n_{y}^{2}+n_{z}^{2}\right)}{\left(\frac{1}{\sigma_{1}}+\frac{1}{\sigma_{2}}+\frac{1}{\sigma_{3}}\right)}
$$

\subsection{Experimental Validation}

Consider an optical tracking system and a calibrated digitizing stylus like the one shown in Figure 1. Suppose that the stylus is oriented so that its $z=0$ plane is perpendicular to the viewing direction of the optical tracker (i.e., directly facing the tracker).

In our simulations, we rotated the stylus about its $x$ axis from $-45^{\circ}$ to $45^{\circ}$ in increments of $15^{\circ}$. At each angle of rotation, we generated 10,000 sets of measured marker locations for the CRF where each measured marker location $\mathbf{g}_{j}$ was the model marker location $\mathbf{f}_{j}$ rotated by the angle of rotation and contaminated with zero-mean, additive Gaussian noise of covariance $\mathbf{N}_{i}=\mathbf{N}=\operatorname{diag}\left(n_{x_{c}}^{2}, n_{y_{c}}^{2}, n_{z_{c}}^{2}\right)$ where $n_{x_{c}}=n_{y_{c}}$ (isotropic noise in the camera viewing plane), $n_{z_{c}}=s n_{x_{c}}$ for some scalar $s \geq 1$ (anisotropic noise in the viewing direction), and $n_{x_{c}}^{2}+n_{y_{c}}^{2}+n_{z_{c}}^{2}=c$ for a constant value $c=0.1^{2}+0.1^{2}+0.3^{2} \mathrm{~mm}^{2}$ (constant total noise magnitude). All noise variances are given in the tracking camera coordinate system. The model marker locations were registered to the noisy measured marker locations using Horn's method (Horn), Ohta and Kanatani's (Ohta) method, and our UKF method (UKFreg). For each registration, we computed TRE using the tip of the stylus as the target. We also computed the expected mean squared TRE for each trial of the UKF method using the calculation described by [9] (UKFest). At each angle, we computed TRE using Equation (3).

We performed the simulations with two different stylus CRFs. The first CRF was identical to that shown in Figure 1. The second CRF also used four markers but in a tetrahedral arrangement; the marker coordinates in units of millimeters were $[45,25,0]^{T}$, $[0,-50,0]^{T},[-45,25,0]^{T}$, and $[0,0,50]^{T}$. 


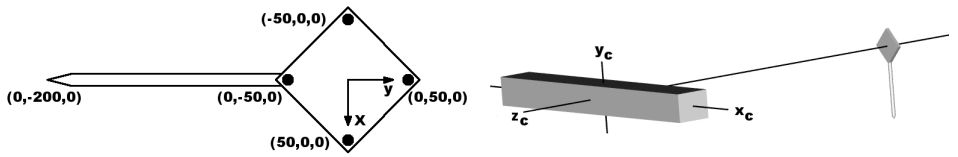

Fig. 1. (Left) Model of the pointing stylus used in our simulations; all units are in millimeters. (Right) Optical tracking system and stylus orientation used in our simulations. Measurement noise variance in the viewing direction $-\mathbf{z}_{c}$ is typically greater than those in the viewing plane.
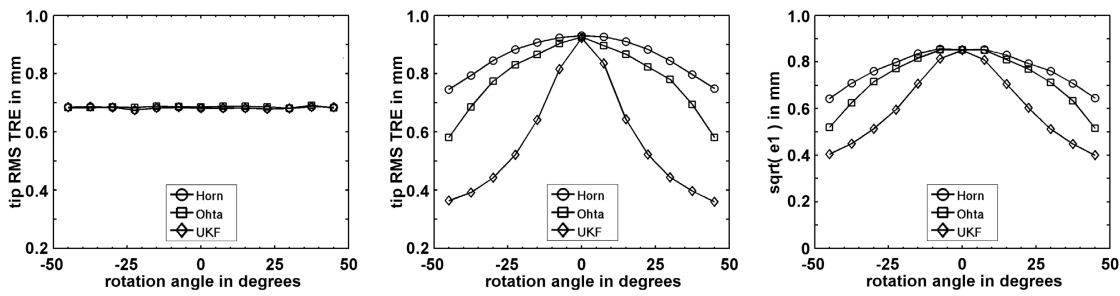

Fig. 2. Simulation results for CRF 1. (Left) TRE versus rotation angle for isotropic noise, $s=1$. (Middle) TRE versus rotation angle for anisotropic noise, $s=5$. (Right) Square root of the largest principal component of the tip error covariance matrix for anisotropic noise $s=3$.

We also performed registration simulations based on identifying fiducials in CT images where the slice spacing is much greater than the pixel size. The Ilizarov frame registration problem described by Ma and colleagues [13] was studied. Only the proximal ring was used, to which we applied a $30^{\circ}$ rotation about the $x$-axis. We used uniformly distributed noise in the range of $[-0.5,0.5] \mathrm{mm}$ for the $x$ and $y$ directions and $[-2.5,2.5]$ for the $z$ direction. TRE was computed on a regular grid with $20 \mathrm{~mm}$ spacing in the plane of the ring.

\section{Results}

The RMS TRE as a function of the stylus rotation angle is shown in Figure 2 for CRF 1. Note that the TRE curves are identical and constant for isotropic noise; the RMS value of $0.68 \mathrm{~mm}$ matches the value predicted by West and Maurer [6, Equation (16)].

We computed the covariance of the tip registration error vector $\boldsymbol{p}_{\theta}-\left(\hat{\mathbf{R}}_{i} \mathbf{p}_{0}+\hat{\mathbf{t}}_{i}\right)$ where $\boldsymbol{p}_{\theta}$ is the true position of the tip at rotation angle $\theta, \hat{\mathbf{R}}_{i}$ and $\hat{\mathbf{t}}_{i}$ are the estimated rotation and translation for trial $i$, and $\mathbf{p}_{0}=[0,-200,0]^{T}$ is the model tip location in millimeters. We then computed the eigenvalues of the covariance matrix (the decorrelated variances); the square root of the two largest eigenvalues (the two largest "standard deviations") as a function of rotation angle are also shown in Figure 2.

The RMS TRE computed from the simulation using UKFreg, UKFest, and Equation (3) are shown in Figure 3 . UKFest tended to overestimate TRE, whereas Equation (3) predicted a value that agreed surprisingly well with the simulation results of UKFreg. We computed the confidence intervals for the RMS TRE values of UKFreg; we used the $\mathrm{BC}_{a}$ bootstrap [14] to deal with the asymmetry of the TRE distributions. 

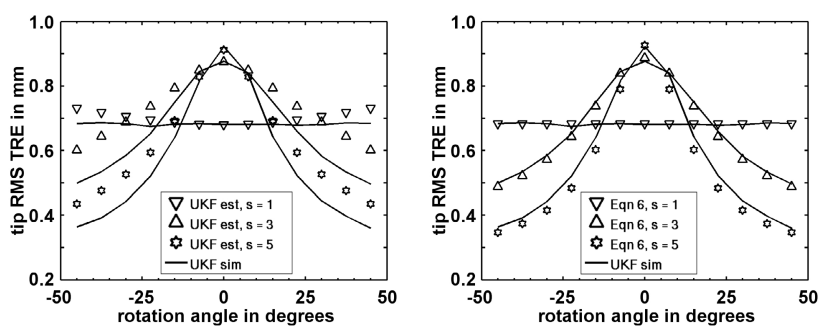

Fig. 3. Root mean squared TRE versus stylus rotation angle for CRF 1. (Left) TRE estimated using UKFest and simulated TRE values. (Right) TRE estimated using Equation (3) and simulated TRE values.
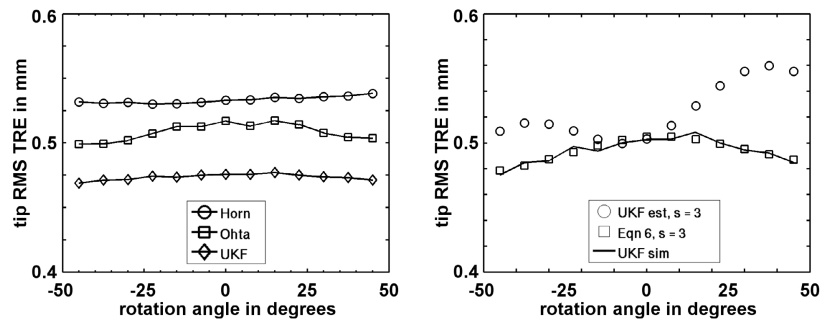

Fig. 4. Root mean squared TRE versus stylus rotation angle for CRF 2. (Left) Simulated values of RMS TRE for $s=3$. (Right) Predicted values of RMS TRE.
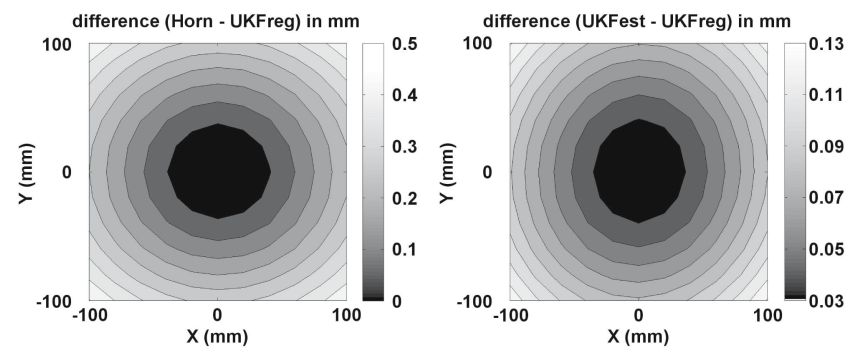

Fig. 5. Ilizarov frame experiment results. (Left) Difference in TRE between Horn's method and UKFreg. (Right) Difference in TRE between UKFest and UKFreg.

We found that the value given by Equation (3) was always within the $95 \%$ confidence interval for isotropic noise. For anisotropic noise, Equation (3) gave a value that was slightly below the lower limits of the confidence intervals; however, the difference between the RMS value and Equation (3) was never more than 7\% of the RMS value.

The results for the second stylus CRF configuration are shown in Figure 4 . The TRE behavior for this stylus was quite uniform over the range of rotation angle. UKFreg had the best TRE performance, but there was dramatically less difference between the registration algorithms. 


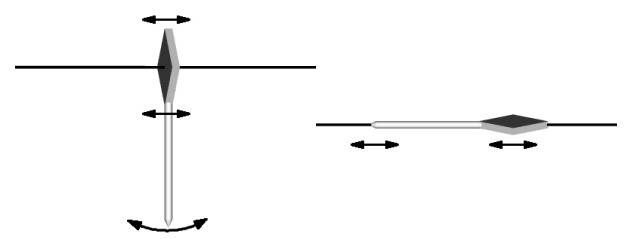

Fig. 6. (Left) Tip TRE is worst when the stylus is oriented to face the direction of greatest noise anisotropy (typically the viewing direction of the camera) because such an orientation results in the greatest expected rotational error. (Right) Tip TRE is minimized by orienting the stylus face away from the camera viewing direction which minimizes the contribution of the rotational error.

The results for the Ilizarov frame example are shown in Figure 5 UKFreg always produced a smaller RMS TRE value than Horn's method. UKFest predicted the simulation results of UKFreg to within $12 \%$ of the RMS TRE value even though we used uniformly distributed noise.

\section{Discussion and Conclusion}

All three estimators used in the simulations had identical worst performances when the CRF of the stylus was directly facing the tracking system (rotation angle of $0^{\circ}$ ). This result is easily explained with reference to Figure 6 Aligning the CRF to face the tracking system produces the situation that allows for the greatest expected rotational error which causes a displacement of the apparent tip location that is proportional to the length of the stylus. Clearly, the stylus orientation that produces the least expected rotation error is that where the face of the $\mathrm{CRF}$ is perpendicular to the viewing direction; of course, in practice the CRF would not be visible to the camera in this orientation.

Horn's method, which assumes isotropic noise, performed the worst in our simulations. Ohta and Kanatani's method, which is optimal with respect to their definition of rotation covariance, performed better than Horn's method but not as well as our UKFreg algorithm; also, it did not produce results consistent with our theoretical prediction given by Equation (3). We are continuing to investigate the cause of this discrepancy.

We implemented the method, UKFest, described by Moghari and Abolmaesumi [9]10] to estimate TRE. UKFest uses the state covariance estimate of the UKF to predict TRE. We found that it overestimates TRE when using a small number of fiducials. This result was not surprising because the estimated covariance is unlikely to be very accurate given the small number of fiducials and the single step update we used.

We were pleasantly surprised by the degree of similarity between the predicted RMS TRE of Equation (3) and the simulated RMS TRE of our UKF algorithm. The superior performance of our algorithm in terms of TRE versus the other algorithms we tested combined with the strong agreement to Equation (3) leads us to speculate that our algorithm is almost optimal under conditions of identically distributed, anisotropic noise. Note that UKFreg does not differ substantially from the EKF algorithm of [4]. Arguably, the UKF is easier to implement because it does not require the computation of Jacobians. We have demonstrated that UKFreg achieves the TRE predicted by our stiffness model, and we expect that the EKF algorithm would perform similarly. 
The simulations using the CRF with one out of plane marker demonstrated the superiority of this design over the flat CRF. West and Maurer [6] showed that a regular tetrahedron was the ideal configuration of fiducials for isotropic noise. Our results show that such a configuration is also preferred over a flat CRF for anisotropic noise.

We have studied the case where a registration algorithm is used to match a model of a target to the measurements made by a tracking system. An alternative method is to use a Kalman-type filter to perform the tracking, which removes the need for an explicit registration algorithm. The UKF we used is easily adapted to such a purpose.

In summary, we have demonstrated that anisotropic noise can have a significant effect on TRE, especially when a suboptimal registration algorithm is used. Our registration algorithm works well in the presence of anisotropic noise, and it produces results consistent with a theoretical model of fiducial registration TRE.

\section{References}

1. Khadem, R., Yeh, C.C., Sadeghi-Tehrani, M., Bax, M.R., Johnson, J.A., Welch, J.N., Wilkinson, E.P., Shahidi, R.: Comparative tracking error analysis of five different optical tracking systems. Computer Aided Surgery 5, 98-107 (2000)

2. Ohta, N., Kanatani, K.: Optimal estimation of three-dimensional rotation and reliability evaluation. IEICE Transactions on Information and Systems E82-D(11), 1247-1252 (1998)

3. Estépar, R.S.J., Brun, A., Westin, C.F.: Robust generalized total least squares iterative closest point registration. In: Barillot, C., Haynor, D.R., Hellier, P. (eds.) MICCAI 2004. LNCS, vol. 3216, pp. 234-241. Springer, Heidelberg (2004)

4. Pennec, X., Thirion, J.P.: A framework for uncertainty and validation of 3D registration methods based on points and frames. IJCV 25(3), 203-229 (1997)

5. Fitzpatrick, J.M., West, J.B., Maurer, Jr, C.R.: Predicting error in rigid-body point-based registration. IEEE Transactions on Medical Imaging 17(5), 694-702 (1998)

6. West, J.B., Maurer, Jr, C.R.: Designing optically tracked instruments for image-guided surgery. IEEE Transactions on Medical Imaging 23(5), 533-545 (2004)

7. Ma, B., Ellis, R.E.: Analytic expressions for fiducial and surface target registration error. In: Larsen, R., Nielsen, M., Sporring, J. (eds.) MICCAI 2006. LNCS, vol. 4190, pp. 637-644. Springer, Heidelberg (2006)

8. Huang, S., Schimmels, J.M.: The bounds and realization of spatial stiffness achieved with simple springs connected in parallel. IEEE Trans. Robot and Automat. 14(3), 466-475 (1998)

9. Moghari, M.H., Abolmaesumi, P.: A high-order solution for the distribution of tre in rigidbody point-based registration. In: Larsen, R., Nielsen, M., Sporring, J. (eds.) MICCAI 2006. LNCS, vol. 4190, pp. 603-700. Springer, Heidelberg (2006)

10. Moghari, M.H., Abolmaesumi, P.: Comparing the unscented and extended Kalman filter algorithms in the rigid-body point-based registration. In: IEEE EMB, pp. 497-500. IEEE Computer Society Press, Los Alamitos (2006)

11. Horn, B.K.P.: Closed-form solution of absolute orientation using unit quaternions. Journal of the Optical Society of America A 4, 629-642 (1987)

12. Lin, Q., Burdick, J., Rimon, E.: A stiffness-based quality measure for compliant grasps and fixtures. IEEE Transactions on Robotics and Automation 16(6), 675-688 (2000)

13. Ma, B., Simpson, A.L., Ellis, R.E.: Proof of concept of a simple computer-assisted technique for correcting bone deformities. In: Ayache, N., Ourselin, S., Maeder, A. (eds.) MICCAI 2007. LNCS, vol. 4792, pp. 935-942. Springer, Heidelberg (2007)

14. DiCiccio, T.J., Efron, B.: Bootstrap confidence intervals. Statistical Science 11(2), 189-228 (1996) 